\section{Research Square}

Preprints are preliminary reports that have not undergone peer review.

They should not be considered conclusive, used to inform clinical practice, or referenced by the media as validated information.

\title{
Identification of a novel interaction between SMC1 DNA damage repair protein and Escherichia coli 0157: H7 EspF using co-immunoprecipitation combined with mass spectrometry
}

\section{Muqing Fu}

Southern Medical University

Ying Hua

Southern Medical University

\section{Kaina Yan}

Southern Medical University

Jia Li

Southern Medical University

Jiali Wu

Southern Medical University

Jinyue Liu

Southern Medical University

Xiaoxia Li

Southern Medical University

Bao Zhang

Southern Medical University

Wei Zhao

Southern Medical University

Qiwei Zhang

Southern Medical University

Chengsong Wan ( $\nabla$ gzwcs@smu.edu.cn )

Southern Medical University

Research article

Keywords: Enterohemorrhagic Escherichia coli 0157: H7, EspF, SMC1, ColP-MS, protein-protein interactions

Posted Date: March 28th, 2020

DOl: https://doi.org/10.21203/rs.3.rs-19408/v1

License: @ (i) This work is licensed under a Creative Commons Attribution 4.0 International License. Read Full License 


\begin{abstract}
Background: It is known that the enterohemorrhagic Escherichia coli (EHEC) 0157: H7 EspF is a multifunctional effector that triggers several damage processes in the host cells. However, in the process of EHEC 0157: H7 infection, the interaction between EspF, its N-or C-terminus, and host proteins, are still unclear.

Results: In this study, we used co-immunoprecipitation combined with mass spectrometry to screen EspF-interacting proteins. A total of 311 host proteins are detected. The N-terminus of EspF is found to interact with 192 proteins, whereas 205 proteins interact with the C-terminus of EspF. These proteins are mainly involved in RNA splicing, endoplasmic reticulum stress, and a variety of metabolic signaling pathways. We verify here for the first time that SMC1 interacts with EspF and more strongly with its C-terminus, and provide evidence that EspF increases p-SMC1 levels. p-SMC1, known to influence the S-phase cell cycle arrest and usually express during periods of DNA damage. Surprisingly, Mass spectrometry reveals that EspF can also phosphorylate H2AX, suggesting that EspF may directly mediate DNA damage through SMC1 phosphorylation.
\end{abstract}

Conclusion: Taken together, this is the first study describing the interaction between EspF and SMC1. Our work lays a foundation for further research on directly EspF-mediated host cells' DNA damage, apoptosis, and even colorectal carcinogenesis.

\title{
Background
}

Enterohemorrhagic E. coli 0157: H7 is an important foodborne pathogen that causes human diarrhea, hemorrhagic colitis, hemolytic uremic syndrome, and thrombotic thrombocytopenic purpura[1, 2]. Severe cases may be life-threatening. EHEC 0157: H7 uses the T3SS type III secretion system to adhere to the brush border of epithelial cells, and then injects effector proteins into host cells. EspF is one of the most important virulence factors of $A$ / $E$ pathogens [3]. EspF exists in EHEC, enteropathogenic Escherichia coli (EPEC), and Citrobacter rodentium, which are potentially harmful to human. It targets the mitochondria and nucleoli[4, 5], destroys the tight junctions of intestinal epithelial cells [6], leading to the disappearance of intestinal epithelial microvilli, and induces host cell apoptosis [7]. Due to its biological effects, it is known as the "Swiss Army Knife" of bacterial pathogens [8].

The N-terminus of EHEC 0157: H7 EspF (1-73 aa) contains a secretion signal (1-20 aa), a host cell mitochondrial targeting signal (mitochondrial targeting signal, MTS, 1-24 aa) [9], and a nucleus-binding domain (Nucleolar targeting domain, NTD, 21-74aa)[5]. Roxas found that EspF localizes to mitochondria, destroys mitochondrial membrane potential, and activates the apoptotic proteases 3 and 9 . The apoptotic proteases can cleave the epidermal growth factor receptor (EGFR) of host cells, leading to the degradation of EGFR and a dramatic increase in host cell death in the late stages of infection [10]. The C-terminus (73-248 aa) is composed of four highly homologous proline-rich sequences(PRRs), each containing a eukaryotic cell SNX9 (Sorting nexin 9), protein binding site SH3 (Src homology 3) motif, and an N-WASP (Neuronal Wiskott-Aldrich syndrome protein) binding domain, a possible actin-binding motif (ABM) [8, 11]. In addition, the results of Amin shows that EspF has a particular anti-phagocytosis effect. The EspF of EHEC 026: H11 and EPEC 0127: H6 can prevent bacteria from being engulfed by macrophages by the PI3K pathway, while the ability of EspF in 0157: $\mathrm{H7}$ is significantly reduced [12].

Although there has been some research on the interaction between EspF and host proteins, the molecular mechanisms of EspF interaction with host proteins and the impact of these interactions on cell damage and apoptosis are still unclear. The identification of the interaction between EspF and the host is important to elucidate the pathogenic mechanism.

ColP-MS is one of the most widely used high-throughput techniques for discovering protein-protein interactions. In our research, we analyzed the molecular function (MF), biological processes (BP) and cellular components (CC) of the related proteins by Gene Ontology (GO), Kyoto Encyclopedia of Genes and Genomes (KEGG), and Clusters of Orthologous Groups (COG) functional annotations. The STRING online tool was used to analyze interactions between target proteins, and Cytoscape software was used to draw the protein interaction network (PPI). The interactions were validated by immunoprecipitation, and the regions of interaction were identified. Co-localization of EspF and the target proteins was performed by confocal microscopy. Our research produced a protein network map between EspF and the host proteins, laying a foundation for further research on how EspF directly mediates DNA damage in the host cells, even causes colorectal carcinogenesis.

\section{Results}

\section{Isolation of differential bands in EspF / EspF-N / EspF-C groups from cell lysates}

After transfecting pEGFP-EspF, pEGFP-EspF / N, and pEGFP-EspF / C-terminus encoding plasmids into $293 \mathrm{~T}$ cells for 48 hours, the lysis proteins were added to Flag columns and IgG columns for co-immunoprecipitation (Fig. 1). pEGFP-EspF was about 58 kDa, pEGFP-EspF / N was $30 \mathrm{kDa}$, and pEGFP-EspF / C was $55 \mathrm{kDa}$. Compared with the IgG group, between $35-40 \mathrm{kDa}$, there were two differentially expressed bands in the pEGFP-EspF group. at $40 \mathrm{kDa}$, there was a differential band in the pEGFP-EspF / N group. At $130 \mathrm{kDa}$, there was a differential band in the pEGFP-EspF / C group. Besides these bands, the bands at the same position in the lanes of the respective IgG groups were also cut out. The bands were digested by mass spectrometry to detect the interacting proteins, and the Flag group-specific proteins (minus the IgG group proteins) were considered as the putative interacting proteins.

\section{Prediction and analysis of interacting protein with EspF}

A total of 708 proteins were identified in this work, 311, 192, and 205 proteins were detected in the pEGFP-EspF group, pEGFP-EspF / N group, and pEGFP-EspF / C group, respectively. We also performed functional annotation (including GO, Pathway, STRING analysis) to identify proteins. Through these analyses, we attempted to discover essential proteins. 
All possible target proteins that interacted with EspF were loaded into the DAVID database for KEGG pathway annotation and GO enrichment. The threshold was set to ps0.05, and pathways or gene functions with higher counts were analyzed. The top 20 pathways were plotted with Graphpad Prism 6 (Table 1). Analysis of the differential bands at about $38 \mathrm{kDa}$ in the pEGFP-EspF group by $\mathrm{GO}$ annotation analysis revealed that the interacting proteins were involved in 25 biological processes (BPs). Of these, the primary were intracellular processes (12.3\%), metabolic processes (10.9\%), biological regulation (8.8\%), and immune stimulation (6.6\%). Cell Components (CCs) enrichment mainly involved cellular anatomical entities (37.7\%), intracellular (36.6\%), and proteincontaining complex (20.9\%). Molecular Functions (MFs) mostly involved binding (53.6\%) and catalytic activity (22.3\%) (Fig. 2A). Pathway analysis showed that the interacting proteins involved 183 pathways, notably Metabolic pathways (32.6\%), Carbon metabolism (19.6\%), and Biosynthesis of amino acids (13.0\%). (Fig. 2B).

Table 1

The top 20 proteins of the $38 \mathrm{kDa}$ differential band between the EspF group and IgG group.

\begin{tabular}{|c|c|c|}
\hline Rank & Protein symbol & Protein annotation \\
\hline 1 & NP_312577.1 & T3SS secreted effector EspF [Escherichia coli 0157:H7 str. Sakai] \\
\hline 2 & sp|P21796|VDAC1_HUMAN & Voltage-dependent anion-selective channel protein 1 OS = Homo sapiens OX 9606 GN = VDAC1 PE = 1 SV = 2 \\
\hline 3 & sp|000165|HAX1_HUMAN & HCLS1-associated protein X-1 OS = Homo sapiens OX = 9606 GN = HAX1 PE = 1 SV $=2$ \\
\hline 4 & sp|P36542|ATPG_HUMAN & ATP synthase subunit gamma, mitochondrial OS $=$ Homo sapiens $\mathrm{OX}=9606 \mathrm{GN}=$ ATP5F1C PE $=1 \mathrm{SV}=1$ \\
\hline 5 & sp|Q9Y623|MYH4_HUMAN & Myosin-4 OS = Homo sapiens OX $=9606 \mathrm{GN}=\mathrm{MYH} 4 \mathrm{PE}=2 \mathrm{SV}=2$ \\
\hline 6 & sp|Q13347|EIF3I_HUMAN & Eukaryotic translation initiation factor 3 subunit I OS $=$ Homo sapiens $\mathrm{OX}=9606 \mathrm{GN}=\mathrm{EIF} 3 \mathrm{I} \mathrm{PE}=1 \mathrm{SV}=1$ \\
\hline 7 & sp|Q15006|EMC2_HUMAN & ER membrane protein complex subunit 2 OS = Homo sapiens OX = $9606 \mathrm{GN}=\mathrm{EMC} 2 \mathrm{PE}=1 \mathrm{SV}=1$ \\
\hline 8 & sp|Q01449|MLRA_HUMAN & Myosin regulatory light chain 2 , atrial isoform OS $=$ Homo sapiens $\mathrm{OX}=9606 \mathrm{GN}=\mathrm{MYL7} \mathrm{PE}=1 \mathrm{SV}=1$ \\
\hline 9 & sp|P17661|DESM_HUMAN & Desmin OS $=$ Homo sapiens OX $=9606 \mathrm{GN}=\mathrm{DES} P E=1 \mathrm{SV}=3$ \\
\hline 10 & sp|Q9P035|HACD3_HUMAN & Very-long-chain (3R)-3-hydroxyacyl-CoA dehydratase 3 OS = Homo sapiens OX = 9606 GN = HACD3 PE = 1 SV = 2 \\
\hline 11 & sp|P60709|ACTB_HUMAN & Actin, cytoplasmic 1 OS = Homo sapiens OX = 9606 GN = ACTB PE $=1 \mathrm{SV}=1$ \\
\hline 12 & sp|P35613|BASI_HUMAN & Basigin OS $=$ Homo sapiens $\mathrm{OX}=9606 \mathrm{GN}=\mathrm{BSG} \mathrm{PE}=1 \mathrm{SV}=2$ \\
\hline 13 & sp|P60891|PRPS1_HUMAN & Ribose-phosphate pyrophosphokinase 1 OS = Homo sapiens OX = 9606 GN = PRPS1 PE = 1 SV = 2 \\
\hline 14 & sp|Q07955|SRSF1_HUMAN & Serine/arginine-rich splicing factor $1 \mathrm{OS}=$ Homo sapiens $\mathrm{OX}=9606 \mathrm{GN}=\mathrm{SRSF} 1 \mathrm{PE}=1 \mathrm{SV}=2$ \\
\hline 15 & sp|Q13505|MTX1_HUMAN & Metaxin-1 OS = Homo sapiens OX = 9606 GN = MTX1 PE = $1 \mathrm{SV}=3$ \\
\hline 16 & sp|P05976|MYL1_HUMAN & Myosin light chain $1 / 3$, skeletal muscle isoform OS $=$ Homo sapiens $O X=9606 \mathrm{GN}=\mathrm{MYL1} P E=2 \mathrm{SV}=3$ \\
\hline 17 & sp|Q96FZ7|CHMP6_HUMAN & Charged multivesicular body protein 6 OS $=$ Homo sapiens OX = 9606 GN = CHMP6 PE $=1 \mathrm{SV}=3$ \\
\hline 18 & sp|P62424|RL7A_HUMAN & $60 \mathrm{~S}$ ribosomal protein L7a OS = Homo sapiens $\mathrm{OX}=9606 \mathrm{GN}=\mathrm{RPL7A} P E=1 \mathrm{SV}=2$ \\
\hline 19 & sp|P35232|PHB_HUMAN & Prohibitin OS $=$ Homo sapiens OX $=9606 \mathrm{GN}=\mathrm{PHB}$ PE $=1 \mathrm{SV}=1$ \\
\hline 20 & sp|P10599|THIO_HUMAN & Thioredoxin OS = Homo sapiens OX $=9606 \mathrm{GN}=\mathrm{TXN} P E=1 \mathrm{SV}=3$ \\
\hline
\end{tabular}

We used STRING to analyze the target proteins with which EspF interacted, and found that RPS6, RPL14, and EIF2S1 had the highest connectivity (Fig. 2C). RPS6 plays an essential role in controlling cell growth and proliferation by selectively translating specific kinds of mRNAs [13]. RPL14 is a large ribosomal subunit component that plays a role in mRNA catabolism and translation [14]. EIF2S1 works in the early stages of protein synthesis by forming a ternary complex with GTP and initiator tRNA [15]. This analysis showed that in addition to gene transcription regulation and protein synthesis, EspF also plays a crucial role in cell proliferation, and catabolism.

The GO annotation analysis of the $36 \mathrm{kDa}$ differential band showed that the interacting proteins were involved in $26 \mathrm{BPs}$, such as intracellular processes (12.5\%), metabolic processes (10.2\%), and biological regulation (9.0\%). CCs and MFs results were consistent with the $38 \mathrm{kDa}$ differential band results (Fig. $3 \mathrm{~A}$ ) Pathway analysis showed that the interacting proteins involved 150 pathways, including Metabolic pathways (23.0\%), Protein processing in the endoplasmic reticulum (13.1\%), and Parkinson disease (11.5\%) (Fig. 3B). STRING analysis showed that the RPL7A, RPS20, and EIF2S1 had a high degree of connectivity, and we found that there was also a high degree of connectivity between the MDH2 and GOT2 (Fig. $3 \mathrm{C}$ ). Among them, MDH2 plays a role in cell metabolism and amino acid acetylation [16]. GOT2 is necessary for metabolite exchange between mitochondria and cytoplasm and plays a crucial role in amino acid metabolism [17].

\section{Prediction and analysis of interacting proteins with EspF-N or C-terminus}

The GO annotation analysis of the differential bands at about $40 \mathrm{kDa}$ in the pEGFP-EspF / $\mathrm{N}$ group showed that the interacting proteins involved $25 \mathrm{BPs}$, such as intracellular processes (12.3\%), metabolic processes (10.8\%), and biological regulation (9.2\%). The CCs and MFs analyses were similar to EspF ( $p E G F P$ EspF). Pathway analysis revealed 228 interacting proteins that were mainly involved in Metabolic pathways (27.1\%), Protein processing in the endoplasmic 
reticulum (9.41\%), and pathways in cancer (8.2\%). (Fig. 4A and B). STRING analysis of target proteins interacting with EspF-N terminus also found that RPL8, RPS9, and EIF3I proteins had the highest degree of connectivity, indicating that EspF may use its N-terminus for ribosomes recognize binding sites (Fig. 4C).

GO annotation analysis of the pEGFP-EspF / C group in the $130 \mathrm{kDa}$ differential band showed that BPs enrichment mostly involved translation (23.1\%), oxidation-reduction process (15.4\%), and intracellular protein transport (13.5\%). CCs enrichment mainly involved intermediate filament (21.3\%), ribosome (13.5\%). MFs mostly involved protein binding (22.4\%), and ATP binding (18.6\%). COG analysis showed that interacting proteins mostly involved translation, ribosomal structure, biogenesis, posttranslational modification, protein turnover, and chaperones (Fig. 5A). KEGG showed that most of the cellular processes involved protein transport, and the signal generation that affected cell growth and apoptosis. Of the interacting proteins, $30.3 \%$ were localized in the cytoplasm, and $27.27 \%$ were localized in the nucleus (Fig. 5BC). The Venn diagram showed 93 proteins that were all involved in the above four pathways (Fig. 5E). Among these proteins, TUBB, and ANXA2 showed strong interaction with EspF-C. TUBB, a main component of tubulin, has GTPase activity, and plays a key role in the microtubule cytoskeleton organization [18]. Studies have shown that EspF interacts with SNX9 in the cytoplasm to induce the formation of membrane tubules and the host cell membrane change [19]. In addition to the SNX9 binding site, EspF can also activate N-WASP to induce actin polymerization, TJ disruption, and anti-phagocytosis [20]. Moreover, EspF also directly binds to cytokeratin 18, which contributes to the collapse of the cytoskeleton[21]. Microfilaments, microtubules, and intermediate fibers together make up the cytoskeletal system. Therefore, we speculated that EspF might also cause cytoskeleton rearrangement by binding TUBB .

In our previous research, ANXA6 and EspF were confirmed to interact[22]. This time we found ANXA2, a member of the ANXA protein family, which is a calcium-regulated membrane-bound protein. ANXA2 participates in the host heat stress response by interacting with Hsp90 [23]. Meanwhile, EspF may regulate calcium ion accumulation and calcium channel protein activity by interacting with ANXA2.

In addition, we also found the SMC family proteins. The interaction between EspF and SMC1 is also found in our previous research and involves multiple signaling pathways [22]. It is understood that SMC1 is an adhesion protein that plays a role in DNA replication and cohesion of sister chromatids. It involves in chromosome dynamics, cell cycle regulation, cell proliferation, and genome stability [24]. Furthermore, when cells were stimulated by DNA damage, SMC1 can be phosphorylated by ATM or ATR to participate in DNA repair and acted as a downstream effector in the ATM / NBS1 branch and the ATR / MSH2 branch to active S-phase checkpoint [25]. Hence, we hypothesized EspF could mediate cellular DNA damage repair by phosphorylating SMC1.

SMC1 was identified as a novel EspF-interacting protein, and its interaction was more robust with the EspF-C terminus

We are the first to study the mechanism of interaction between EspF and DNA damage repair proteins. Western blotting and confocal analysis further confirmed the interaction between EspF and SMC1. Co-immunoprecipitation results confirmed that EspF interacted with SMC1, and the interaction was stronger when with EspF-C terminus (Fig. 6A). Immunofluorescence analysis also showed that EspF and SMC1 were co-localized in the cytoplasm (Fig. 6B), and EspF relocated SMC1 more from the nucleolus into the cytoplasm, suggesting that SMC1 may not play its usual role in the nucleolus.

To investigate the EspF-SMC1 interplay, we measured SMC1 and its phosphorylation levels after Caco2 cells were transfected with EspF. Immunofluorescence showed that EspF could significantly increase the expression of p-SMC1, and p-SMC1 was distinctly localized in the cytoplasm and co-localized with EspF (Fig. 6C). Compared with the control, the level of SMC1 remained unchanged after transfection with EspF, but p-SMC1 significantly increased (Fig. 6D-E). We then verified the results by infecting HT-29 cells with the strain. The expression of p-SMC1 in cells transfected with the EDL 933 was higher than infected $\triangle \mathrm{espF}$ strain, and infecting the complement $\triangle \mathrm{espF} / \mathrm{pespF}$ restored p-SMC1 expression. The above results verified that EspF could increase the expression level of p-SMC1.

In general, when cell DNA is damaged, cyclin-mediated cell cycle arrest will occur. During this period, DNA damage repair proteins are recruited to double sideband break (DSB) [26]. Therefore, we speculated that EspF might lead to DNA damage, which stimulates the S-phase detection point by increases p-SMC1 expression, thus mediating damage repair.

\section{EspF may mediate DNA damage by modifying the histones}

Mass spectrum results showed that EspF could modify multiple sites of various proteins (Table 2). Among the modification results, SFXN1, HAX1, ElF3I, ATG16L1, and DNA damage binding proteins had high scores. SFXN1 is a mitochondrial serine transporter that mediates serine into mitochondria and plays an essential role in the single-carbon metabolic pathway[27]. EspF can cause oxidative phosphorylation and methylation of SFXN1, which may mediate the metabolism required component for transport in and out of the mitochondria. 
Table 2

EspF-mediated phosphorylation, acetylation, and methylation of the host proteins.

\begin{tabular}{|c|c|c|c|c|c|c|}
\hline & Protein symbol & Score & $\begin{array}{l}\text { Pep } \\
\text { before }\end{array}$ & Pep_seq & $\begin{array}{l}\text { Pep } \\
\text { after }\end{array}$ & Pep mod \\
\hline 1 & sp|Q9H9B4|SFXN1_HUMAN & 270 & $\mathrm{R}$ & ILMAAPGMAIPPFIMNTLEK & $\mathrm{K}$ & 2 Oxidation (M); Phospho (ST); Methyl (K) \\
\hline 2 & sp|P09651|ROA1_HUMAN & 267 & $\mathrm{~K}$ & SESPKEPEQLR & $\mathrm{K}$ & Phospho (ST) \\
\hline 3 & sp|P05388|RLA0_HUMAN & 187 & $\mathrm{~K}$ & EDLTEIR & $\mathrm{D}$ & Phospho (ST) \\
\hline 4 & sp|P05388|RLA0_HUMAN & 187 & $\mathrm{R}$ & GNVGFVFTK & $E$ & Phospho (ST); Acetyl (K) \\
\hline 5 & sp|P05388|RLA0_HUMAN & 187 & $\mathrm{~K}$ & CFIVGADNVGSK & Q & Phospho (ST) \\
\hline 6 & sp|A0A075B6P5|KV228_HUMAN & 172 & $\mathrm{R}$ & FSGSGSGTDFTLK & I & Phospho (ST) \\
\hline 7 & sp|000165|HAX1_HUMAN & 145 & $\mathrm{~K}$ & ITKPDGIVEERR & $\mathrm{T}$ & Phospho (ST); Acetyl (K) \\
\hline 8 & sp|Q13347|EIF3I_HUMAN & 115 & $\mathrm{~K}$ & QLALLKTNSAVR & $\mathrm{T}$ & Phospho (ST); Acetyl (K) \\
\hline 9 & sp|Q01449|MLRA_HUMAN & 112 & $\mathrm{~K}$ & VSVPEEELDAMLQEGK & G & Phospho (ST); Methyl (K) \\
\hline 10 & sp|P35613|BASI_HUMAN & 89 & $\mathrm{~K}$ & GSDQAIITLRVR & S & 2 Phospho (ST); Methyl (R) \\
\hline 11 & sp|P11908|PRPS2_HUMAN & 89 & $\mathrm{~K}$ & IASSSRVTAVIPCFPYAR & Q & $\begin{array}{l}3 \text { Phospho (ST); Phospho (Y); } 2 \text { Methyl } \\
\text { (R) }\end{array}$ \\
\hline 12 & sp|P60891|PRPS1_HUMAN & 78 & $\mathrm{~K}$ & IASASRVTAVIPCFPYAR & Q & Phospho (ST); Phospho (Y); Methyl (R) \\
\hline 13 & sp|Q13505|MTX1_HUMAN & 72 & $\mathrm{~K}$ & YNADYDLSAR & Q & Phospho (Y) \\
\hline 14 & sp|P35232|PHB_HUMAN & 70 & $\mathrm{R}$ & SRPRNVPVITGSK & $\mathrm{D}$ & Phospho (ST); Methyl (R); Acetyl (K) \\
\hline 15 & sp|Q99880|H2B1L_HUMAN & 65 & $\mathrm{~K}$ & AVTKYTSSK & - & Phospho (ST); Phospho (Y); 2 Methyl (K) \\
\hline 16 & sp|Q92522|H1X_HUMAN & 64 & $\mathrm{~K}$ & AAKPSVPK & V & Phospho (ST); Methyl (K); Acetyl (K) \\
\hline 17 & sp|Q86V81|THOC4_HUMAN & 58 & - & MADKMDMSLDDIIK & $\mathrm{L}$ & 2 Oxidation (M); Phospho (ST); Methyl (K) \\
\hline 18 & sp|P0C0S8|H2A1_HUMAN & 48 & $\mathrm{~K}$ & KTESHHK & A & Phospho (ST); Acetyl (K) \\
\hline 19 & sp|P16104|H2AX_HUMAN & 48 & $\mathrm{~K}$ & TSATVGPKAPSGGK & $\mathrm{K}$ & 2 Phospho (ST); Methyl (K) \\
\hline 20 & sp|Q8IUE6|H2A2B_HUMAN & 48 & $\mathrm{~K}$ & KTESHKPGK & $\mathrm{N}$ & Phospho (ST); Methyl (K); 2 Acetyl (K) \\
\hline 21 & sp|Q9Y375|CIA30_HUMAN & 48 & $\mathrm{R}$ & KFSKPTSALYPFLGIR & $\mathrm{F}$ & Phospho (ST); Acetyl (K) \\
\hline 22 & sp|014983|AT2A1_HUMAN & 46 & $\mathrm{~K}$ & EYEPEMGKVYR & $A$ & Oxidation (M); Phospho (Y) \\
\hline 23 & sp|Q9Y394|DHRS7_HUMAN & 46 & $\mathrm{~K}$ & LGVSLVLSAR & $\mathrm{R}$ & Phospho (ST); Methyl (R) \\
\hline 24 & sp|P08758|ANXA5_HUMAN & 45 & $\mathrm{R}$ & VMVSRSEIDLFNIR & $\mathrm{K}$ & Oxidation (M); Phospho (ST) \\
\hline 25 & sp|Q5JTV8|TOIP1_HUMAN & 41 & $\mathrm{~K}$ & TPQEWAPQTAR & I & 2 Phospho (ST) \\
\hline 26 & sp|P10412|H14_HUMAN & 40 & $\mathrm{~K}$ & ATGAATPK & $\mathrm{K}$ & Phospho (ST) \\
\hline 27 & sp|P16402|H13_HUMAN & 40 & $\mathrm{~K}$ & KAASGEGKPK & $A$ & Phospho (ST) \\
\hline 28 & sp|P22492|H1T_HUMAN & 40 & $\mathrm{~K}$ & KPRATTPK & $\mathrm{T}$ & Phospho (ST); Acetyl (K) \\
\hline 29 & sp|Q02539|H11_HUMAN & 40 & $\mathrm{~K}$ & KPKTVKPK & $\mathrm{K}$ & Phospho (ST) \\
\hline 30 & sp|P16403|H12_HUMAN & 40 & $\mathrm{~K}$ & AGGTKPK & $\mathrm{K}$ & Phospho (ST); Methyl (K) \\
\hline 31 & sp|Q9UFE4|CCD39_HUMAN & 37 & $\mathrm{R}$ & SPSHTSLSAR & S & Phospho (ST) \\
\hline 32 & sp|Q9Y4L1|HYOU1_HUMAN & 37 & $\mathrm{~K}$ & FTKPRPRPK & $\mathrm{D}$ & Phospho (ST); Methyl (K) \\
\hline 33 & sp|P31944|CASPE_HUMAN & 36 & $\mathrm{R}$ & LALILCVTK & $A$ & Phospho (ST); Acetyl (K) \\
\hline 34 & sp|P07355|ANXA2_HUMAN & 36 & $\mathrm{R}$ & KGTDVPK & W & Phospho (ST); Acetyl (K) \\
\hline 35 & sp|P07355|ANXA2_HUMAN & 36 & $\mathrm{~K}$ & GTDVPKWISIMTER & $S$ & $\begin{array}{l}\text { Oxidation (M); Phospho (ST); Methyl (R); } \\
\text { Acetyl (K) }\end{array}$ \\
\hline 36 & sp|P07355|ANXA2_HUMAN & 36 & $\mathrm{~K}$ & GTDVPKWISIMTER & S & Oxidation (M); 2 Phospho (ST); Methyl (R) \\
\hline 37 & sp|P07355|ANXA2_HUMAN & 36 & $\mathrm{~K}$ & LSLEGDHSTPPSAYGSVK & $A$ & Phospho (ST); Methyl (K) \\
\hline 38 & sp|Q92466|DDB2_HUMAN & 34 & $\mathrm{~K}$ & VTHVALNPCCDWFLATASVDQTVK & I & 3 Phospho (ST) \\
\hline 39 & sp|Q8WV16|DCAF4_HUMAN & 34 & $\mathrm{R}$ & MGFNASSMLRK & $S$ & Phospho (ST); Methyl (K) \\
\hline
\end{tabular}




\begin{tabular}{|llcllll|}
\hline & Protein symbol & Score & $\begin{array}{l}\text { Pep } \\
\text { before }\end{array}$ & Pep_seq & $\begin{array}{l}\text { Pep } \\
\text { after }\end{array}$ & Pep mod \\
\hline 40 & splQ676U5|A16L1_HUMAN & 34 & $\mathrm{~K}$ & CGSDWTR & $\mathrm{V}$ & Phospho (ST) \\
\hline 41 & sp|Q7L5D6|GET4_HUMAN & 31 & $\mathrm{~K}$ & NKSSASVVFTTYYTQK & $\mathrm{H}$ & Phospho (ST); Methyl (K) \\
\hline 42 & sp|P84243|H33_HUMAN & 30 & $\mathrm{~K}$ & SAPSTGGVKKPHR & $\mathrm{Y}$ & 2 Phospho (ST) \\
\hline
\end{tabular}

HAX1 recruits the Arp2 / 3 complex to the cell cortex, and through its interaction with KCNC3, it reconstitutes the cortical actin cytoskeleton[28]. EspF may rearrange the cytoskeleton and modulate cell survival through phosphorylation and acetylation of the HAX1. EIF3I is a part of the EIF-3 complex and uses different RNA stem-loop binding modes to increase ERK translation[29]. EspF may act on the translation of mRNA involved in cell proliferation (including cell cycle, differentiation, and apoptosis) through phosphorylation and acetylation of EIF3I. EspF may also phosphorylate some autophagy-related proteins, including ATG16L1 and PHB, that mediate BPs such as cell autophagy and protein transport[30].

We also discovered that EspF could modify a series of histones, such as Histone H1.3, Histone H2A type 1, and Histone H1x, which are the core components of nucleosomes. Nucleosomes encapsulate DNA into chromatin, limiting DNA's entry into cellular mechanisms[31]. Therefore, histone modifications play a central role in transcription regulation, DNA damage repair, DNA replication, and chromosomal stability. DNA accessibility is also regulated by a complex set of posttranslational modifications of histones and nucleosome remodeling[32]. EspF may affect epigenetic changes in host cells. Importantly, we found that EspF could phosphorylate histone H2AX, a known marker of DNA damage[33], suggesting that EspF can cause DNA damage.

Next, we discovered that EspF also modified the damage-specific DNA-binding protein 2 (DDB2), a kind of DNA repair protein. DDB2 is originally identified as a DNA damage recognition factor that promotes genomic nucleotide excision repair (GG-NER) in human cells. DDB2 is also involved in other important BPs such as chromatin remodeling, gene transcription, cell cycle regulation, and protein decay. Recently, the potential of DDB2 in the development and progression of various cancers has been described. DDB2 activity occurs in several stages of canceration, including cancer cell proliferation, survival, invasion, and cancer stem cell formation[34]. Previous research showed that EspF could cause cell apoptosis by targeting mitochondria and releasing cytochrome c[4], but it is still unclear whether EspF can mediate DNA damage through nuclear modification of histones in the early period, which lays the foundation for our future research.

\section{Discussion}

Previous studies screened host proteins with EspF interactions mainly through yeast two-hybrid and BiFC fluorescence methods[22, 35]. The former cannot determine whether the interactions exist in mammalian cells, while the latter use tags that may affect the conformation or activity of the mark protein, leading to false-negative results. Therefore, we opted for a classic ColP-MS assay to screen host proteins that interact with EspF in $293 \mathrm{~T}$ cells. MS results identified 311 proteins. We then analyze the BPs, MFs, and CCs of the interacting proteins by GO enrichment, KEGG pathway, and STRING analyses.

MS results showed that host proteins interacting with EspF were mostly involved in metabolic pathways and localized in the cytoplasm and nucleolus. We found that EspF interacted strongly with ribosomal RPL, RPS, and EIF family proteins, and EspF can phosphorylate them. Previous proteomics studies have shown that many ribosomal protein levels in intestinal cells decrease after EPEC infection[36]. In cells expressing EspF, pre-rRNA synthesis is blocked. At the same time, EspF-dependent EPEC infection reduces the expression level of ribosomal protein RPL9, and changes the relocation of RPS5 and U8 small nucleolar RNA (snoRNA)[37]. Our results provided further support to the hypothesis that EspF may exercise its biological function by regulating ribosomal protein synthesis and relocation. Future research will attempt to decipher the mechanism by which EspF leads to the inhibition of ribosome synthesis.

EspF targets mitochondria and regulates the expression of DNA mismatch repair proteins in host cells through post-transcriptional manipulation, leading to depletion of Apc and MMR proteins in host cells[38], and increases the instability of microsatellite DNA sites, which is a precursor to DNA damage and can even lead to colon cancer[39]. In this work, we also found that EspF can lead to H2AX phosphorylation and modify DDB2. Phosphorylated H2AX is an indicator of DNA damage. This series of evidence suggests that when EspF enters the host cell, it may cause DNA damage in the early stage by regulating the expression of damage repair proteins. SMC1 is a chromosomal structural protein. When DNA is damaged, the upstream ATM will phosphorylate SMC1 in DSB to form the BRCA1-NBS1-MRE11 complex, and SMC1 activates the S-phase checkpoint in DNA damage repair[40]. Abnormal gene expression or mutation can lead to a deficient DNA damage repair pathway, which are closely related to tumorigenesis[41]. Thus, we focused on the interaction between EspF and SMC1. Immunofluorescence and co-immunoprecipitation results confirmed the interaction, and the interaction with EspF-C was stronger than with EspF-N terminus.

Furthermore, EspF can increase the expression of p-SMC1 in $\mathrm{Caco}_{2}$ cells and localized it in the cytoplasm. Thus, EspF may mediate DNA damage repair, cell proliferation, and even canceration by phosphorylating SMC1 (Fig. 7). The DNA damage effect also needs to be study by transfecting siSMC1 or adding ATMi, which is an inhibitor of ATM. There is already the evidence that A/E E. coli infects intestinal epithelial cells, causing DNA damage in host cells, and accelerates the development of cancer through the toll-like receptor signaling pathway, NF-KB pathway, and other cellular inflammation pathways[42]. However, there is no research on whether EspF can directly cause DNA damage, which is worthy of further study.

Meanwhile, we separately studied the interacting proteins and pathogenic mechanisms of EspF-N/C terminus. We found that the proteins interacting with the $\mathrm{N}$-terminus are mostly involved in metabolic pathways and protein processing in the endoplasmic reticulum. Our previous research proved that the $\mathrm{N}$-terminus is essential for cell apoptosis, inflammatory response, and animal toxicity[43]. Thus, we speculate that EspF-N may also mediate apoptosis through endoplasmic reticulum stress. Previous studies have shown that the EspF-C terminus can bind to various host protein motifs. We were the first to find that the EspF-C terminus can interact with the SMC1, a DNA damage repair protein, which provides a new basis for future studies on DNA damage, microsatellite instability, and even colon cancer caused by EspF. 
It is undeniable that ColP-MS has certain limitations in the screening process. First, the bands for mass spectrometry should be distinct, and some weak interactions may not be detected. Second, different levels of protein expression between different cells may affect the binding efficiency of coimmunoprecipitation. Third, ColP-MS detects transient effects, so it is not possible to screen all host proteins that interact with EspF. We need further experiments to verify the real interactions.

The host proteins interacting with EspF screened by our research may directly interact with EspF or form multiple subcomplexes with EspF. Furthermore, we confirmed that EspF interacted with SMC1, most likely through its $C$ terminus. At the same time, EspF can increase the p-SMC1 expression level and relocate $\mathrm{p}$ SMC1 into the cytoplasm. This provides us with new insights into the role of EspF in mediating DNA damage repair. Further research is needed on what processes EspF is involved in and how these effects mediate the bacterial-host pathogenesis. Our screening provides directions for future analysis of the potential biological role played by EspF and its $\mathrm{N}$ and $\mathrm{C}$ terminus.

\section{Conclusion}

In this study, we focused on 311 host proteins that interact with the EHEC 0157: H7 EspF and used bioinformatics enrichment to analyze their molecular functions, BPs, and cellular pathways. These findings provide new candidates for EspF interactions, suggesting that EspF can phosphorylate $\mathrm{H} 2 \mathrm{AX}$ and regulate the DNA damage repair process by interacting with SMC1. These results are very encouraging, and we provided a PPI network of interactions of EspF with the host, which brings great hope for the field of protein interactions mediating the pathogen EHEC 0157: H7 and host cell interplay.

\section{Methods \\ Cell lines and strains}

293T, $\mathrm{CaCO}_{2}$, and Vero cells were cultured overnight in DMEM (Gibco) containing $10 \%$ fetal bovine serum (FBS), $1 \%$ penicillin and streptomycin at $5 \%$ CO ${ }_{2}$ and $37{ }^{\circ} \mathrm{C}$. HT-29 cells were cultured overnight in RPMI- 1640 (Gibco) medium containing $10 \% \mathrm{FBS}$ and $1 \%$ penicillin and streptomycin at $5 \% \mathrm{CO}_{2}$ and $37{ }^{\circ} \mathrm{C}$. The pEGFP-N1 plasmid, strain EHEC 0157: H7 EDL 933, $\Delta$ espF, and $\triangle$ espF / pespF were stored in our laboratory[43]. DH5a competent cells and LA high-fidelity enzyme were purchased from TaKaRa. Restriction enzymes EcoR I and BamH I were purchased from Thermo Fisher Scientific. The primers used in this study were synthesized by Sangon Biotech (Shanghai, China). Gene sequencing was performed by Guangzhou IGE Biotechnology.

\section{Construction of pEGFP-EspF, pEGFP-EspF / N, and pEGFP-EspF / C-terminus encoding plasmids}

We amplified the espF gene and its N-terminus (1-219 bp) and C-terminus (220-747 bp) with LA high-fidelity enzyme on the genome of EDL 933 . Each constructed plasmid was labeled with 3xFlag. Primer espF-F: CCGGAATTCGCCACCATGCTTAATGGAATTAGTAACGCTG

and espF-R:

CGCGGATCCCCGCTACCGCCGCTTCCCTTGTCCTTATCGTCGTCATCCTTGTAATCCTTATCGTCGTCATCCTTGTAATCCTTATCGTCGTCATCCTTGTAATCCCCTTTC amplified the espF gene; primer espF / N-F: CCGGAATTCGCCACCATGCTTAATGGAATTAGACAC

and espF / N-R: CGCGGATCCCCACCAGAGCCACCCTTATCGTCGTCATCCTTGTAATCGGGAGTAAATGAAGTCACCTG amplified the espF / N; primer espF / C-F: CCGGAATTCGCCACCATGTCTCGTCCGGCACCGCGCGCCCCCACC and espF / C-R: CGCGGATCCCCACCTCCCC amplified the espF /C. The PCR product was digested with EcoRI / BamH I restriction enzyme and T-linked to the pEGFP-N1 plasmid to generate pEGFP-EspF, pEGFP-EspF / N, and pEGFP-EspF / C plasmids. Sequencing verified the integrity of the constructed plasmids.

293T cells were cultured according to standard methods, and the cells were plated on $10 \mathrm{~cm}^{2}$ culture dishes (NEST, Hong Kong, China). According to the manufacturer's instructions, the cells were transfected with pEGFP-EspF, pEGFP-EspF / N, pEGFP-EspF / C plasmids using Lipofectamine 3000 (Thermo, USA).

\section{ColP and Western Blot}

We followed the instructions of the co-immunoprecipitation Pierce ColP Kit 26149 . We took $80 \mu \mathrm{l}$ of the control agarose resin slurry and added 1 mg of the protein sample to remove the non-specifically bound proteins. Then we took $20 \mu$ of AminoLink resin slurry into the spin column, added $15 \mu \mathrm{g}$ IgG antibody (Abclonal)/Flag antibody (CST), and co-incubated the mixture for $1 \mathrm{~h}$. The non-specifically bound protein was added to the spin column, left at $4{ }^{\circ} \mathrm{C}$ overnight, washed three times with IP lysis, and then eluted the Flag / IgG protein complex with the eluent. The above samples were used for 10\% SDS-PAGE electrophoresis for immunoblotting. The primary antibodies of rabbit anti-Flag (CST) and rabbit anti-SMC1 (CST) were added and incubated overnight at $4{ }^{\circ} \mathrm{C}$. After three washes with TBST, the corresponding secondary antibody was added, incubated at room temperature for 1 hour, and the bands were visualized using an ECL chemical solution.

\section{Silver stain}

We took out the polyacrylamide gel after electrophoresis, added $20 \mathrm{ml}$ of fixing solution, and left the gel for 15 min on a shaker under gentle agitation. Twenty $\mathrm{ml}$ of sensitizer was added, and the gel was left under gentle agitation for $45 \mathrm{~min}$. Then we added the staining solution for $30 \mathrm{~min}$. After rinsing with pure water, a chromogenic solution was added, and the protein bands became evident. The chromogenic solution was quickly poured out, and a stop solution was added. We then cut out the different bands, added the elimination solution, and shook the samples. After confirming that the slice was colorless, we sent it to Huijun Biological Company (Guangzhou) for mass spectrometry. 
The decolorizing solution was added to the slice, which was then washed with mass spectrometry water. The gel was ground, acetonitrile was added, the supernatant was removed, $50 \mathrm{ml}$ of the enzymatic hydrolysis was added, and then the sample was incubated at $37^{\circ} \mathrm{C}$ for $6 \mathrm{~h}$. Then, $0.1 \%$ formic acid water was added, and the sample was shaken for $5 \mathrm{~min}$ to make it fully swelled. Acetonitrile was added, and the sample was shaken for another 5 min. The sample was centrifuged, and the supernatant was recovered to be analyzed on a mass spectrometer.

\section{Bacterial infections}

EHEC 0157: H7 EDL 933 was cultured in LB medium, $\triangle \mathrm{espF}$ and $\triangle \mathrm{espF} / \mathrm{pespF}$ in LB broth medium containing kana antibiotics at $37^{\circ} \mathrm{C}$ for 12 hours, and the HT-29 cells were seeded in $10 \mathrm{~cm}^{2}$ culture dishes. When the cells reached $95 \%$ confluence, the cellular monolayer was washed with PBS, and the HT-29 cells were infected with bacteria at an $\mathrm{MOI}=100$ : 1 , and incubated in a humidified incubator at $37{ }^{\circ} \mathrm{C}$ and $5 \% \mathrm{CO}_{2}$ for 6 hours. Then, the medium was aspirated, the cells were gently washed with PBS, and the proteins were collected for immunoblotting.

\section{Immunofluorescence}

Vero cells were plated on a confocal dish (35 mm, NEST, Hong) and grown overnight. When the cells reached $50 \%$ confluence, the cells were transiently transfected with the pEGFP, pEGFP-EspF, pEGFP-EspF / N, and pEGFP-EspF / C vectors. After 48 hours, the cells were gently washed with PBS three times, then fixed in $4 \%$ paraformaldehyde for $15 \mathrm{~min}$ at room temperature, and blocked with $0.1 \%$ Triton X-100 for 30 min. The primary antibody was added, and the sample was incubated at $4{ }^{\circ} \mathrm{C}$ overnight. The sample was washed with PBS three times, incubated with the secondary antibody for 30 min, and then DAPI was added for nuclear staining. Cellular colocalization was observed under an FV1000 confocal microscope (Olympus, Tokyo, Japan).

\section{Bioinformatics Analysis}

The DAVID online tool was used to analyze protein MFs, BPs, CCs, cell localization, and KEGG signaling pathways. Taking the DAVID database as a background reference, we used GO and KEGG enrichment analysis to analyze the interacting proteins. The statistical analysis was based on Fisher's exact test. We used STRING to analyze PPIs. Venn diagrams were used to examine each protein pathway annotation.

\section{Declarations}

\section{Abbreviations}

ColP: co-immunoprecipitation combined with mass spectrometry; BPs: biological processes; CCs: Cell Components; MFs: Molecular Functions; GO: Gene Ontology; KEGG: Kyoto Encyclopedia of Genes and Genomes; COG: Clusters of Orthologous Groups; PPI: protein interaction network

\section{Acknowledgments}

This research is supported by funding from the Natural Science Foundation of Guangdong Province (No.2018B030311063) and co-funded by the Guangzhou Health Care Collaborative Innovation Major Project (No.201704020219), administered by Southern Medical University. The authors have no other relevant affiliations or financial involvement with any organization or entity with a financial interest in or financial conflict with the subject matter or materials discussed in the manuscript

apart from those disclosed. We thank LetPub (www.letpub.com) for its linguistic assistance during the preparation of this manuscript.

\section{Author contributions}

MQF designed the study the research; MQF, YH and KNY conceived the methods; MQF, JL, JLW, JYL and XXL performed the experiments; MQF, BZ, WZ, QWZ and CSW discussed the data and wrote the paper.

\section{Funding}

This research is supported by funding from the Natural Science Foundation of Guangdong Province (No.2018B030311063) and co-funded by the Guangzhou Health Care Collaborative Innovation Major Project (No.201704020219), administered by Southern Medical University.

\section{Availability of data and materials}

The datasets used and/or analyzed during this study available from the corresponding author on reasonable request.

\section{Ethics approval and consent to participate}

Not applicable. 
Consent for publication

Not applicable.

Competing interests

The authors declare that they have no competing interests.

\section{References}

1. Kaper JB: Pathogenic Escherichia coli. NAT REV MICROBIOL 2005, 295(6-7):355-356.

2. Al JMRE: Epidemiology of Escherichia coli 0157:H7 Outbreaks, United States, 1982-2002 - Volume 11, Number 4-April 2005 - Emerging Infectious Disease journal - CDC. 2005, 11(4):603-609.

3. M M, P P: Enterohemorrhagic Escherichia coli as the cause of diarrhea in the Czech Republic, 1965-2013. Epidemiologie, mikrobiologie, imunologie : casopis Spolecnosti pro epidemiologii a mikrobiologii Ceske lekarske spolecnosti J.E. Purkyne 2014.

4. Nougayrède JP, Donnenberg MS: Enteropathogenic Escherichia coli EspF is targeted to mitochondria and is required to initiate the mitochondrial death pathway. CELL MICROBIOL 2004, 6(11):1097-1111.

5. Dean P, Scott JA, Knox AA, Quitard S, Watkins NJ, Kenny B, Van Nhieu GT: The Enteropathogenic E. coli Effector EspF Targets and Disrupts the Nucleolus by a Process Regulated by Mitochondrial Dysfunction. PLOS PATHOG 2010, 6(6):e1000961.

6. Weflen AW, Alto NM, Viswanathan VK, Hecht G: E.coli secreted protein F promotes EPEC invasion of intestinal epithelial cells via an SNX9-dependent mechanism. CELL MICROBIOL 2010, 12(7):919-929.

7. Zhao SZYW: The N-Terminal Domain of EspF Induces Host Cell Apoptosis after Infection with Enterohaemorrhagic Escherichia coliO157:H7. PLOS ONE 2010, 8: e55164.

8. Holmes A, Muhlen S, Roe AJ, Dean P: The EspF effector, a bacterial pathogen's swiss army knife. Infection \& Immunity 2010, 78(11):4445-4453.

9. Charpentier $X$, Oswald E: Identification of the secretion and translocation domain of the enteropathogenic and enterohemorrhagic Escherichia coli effector Cif, using TEM-1 beta-lactamase as a new fluorescence-based reporter. J BACTERIOL 2004, 186(16):5486-5495.

10. Roxas JL, Koutsouris A, Viswanathan VK: Enteropathogenic Escherichia coli-Induced Epidermal Growth Factor Receptor Activation Contributes to Physiological Alterations in Intestinal Epithelial Cells. INFECT IMMUN2007, 75(5):2316-2324.

11. Mcnamara BP, Donnenberg MS: A novel proline-rich protein, EspF, is secreted from enteropathogenic Escherichia coli via the type III export pathway\&nbsp. FEMS MICROBIOL LETT 2006, 166(1):71-78.

12. Tahoun ASGS: Comparative Analysis of EspF Variants in Inhibition of Escherichia coli Phagocytosis by Macrophages and Inhibition of E. coli Translocation through Human- and Bovine-Derived M Cells. INFECT IMMUN 2011, 79(11):4716.

13. Meyuhas O: Ribosomal Protein S6 Phosphorylation: Four Decades of Research. International Review of Cell \& Molecular Biology 2015, 320:41.

14. Enerly E, AHMADI H, SHALCHIAN-TABRIZI K, LAMBERTSSON A: Identification and comparative analysis of the RpL14 gene from Takifugu rubripes. HEREDITAS 2003, 139(2):143-150.

15. Boye E, Grallert B: elF2a phosphorylation and the regulation of translation. CURR GENET 2019(84-98).

16. Gabay-Maskit S, Schuldiner M, Zalckvar E: Validation of a yeast malate dehydrogenase 2 (Mdh2) antibody tested for use in westem blots. F1000research 2018, 7.

17. Yang S, Hwang S, Kim M, Seo SB, Lee J, Jeong SM: Mitochondrial glutamine metabolism via GOT2 supports pancreatic cancer growth through senescence inhibition. CELL DEATH DIS 2018, 9(2):55.

18. Ti S, Alushin GM, Kapoor TM: Human $\beta$-Tubulin Isotypes Can Regulate Microtubule Protofilament Number and Stability. DEV CELL 2018.

19. Marches O, Batchelor M, Shaw RK, Patel A, Cummings N, Nagai T, Sasakawa C, Carlsson SR, Lundmark R, Cougoule C: EspF of Enteropathogenic Escherichia coli Binds Sorting Nexin 9. J BACTERIOL 2006, 188(8):3110-3115.

20. Garber JJ, Mallick EM, Scanlon K, Turner JR, Donnenberg MS, Leong JM, Snapper SB: Attaching-and-Effacing Pathogens Exploit Junction Regulatory Activities of N-WASP and SNX9 to Disrupt the Intestinal Barrier. 2018.

21. Viswanathan VK, Lukic S, Koutsouris A, Miao R, Hecht G: Cytokeratin 18 interacts with the enteropathogenic Escherichia coli secreted protein F (EspF) and is redistributed after infection. CELL MICROBIOL 2004, 6(10):987-997.

22. Hua Y, Ju J, Wang X, Zhang B, Zhao W, Zhang Q, Feng Y, Ma W, Wan C: Screening for host proteins interacting withEscherichia coli 0157:H7 EspF using bimolecular fluorescence complementation. FUTURE MICROBIOL 2018, 13(1):37-58.

23. Díaz-Díaz A, Roca-Lema D, Casas-Pais A, Romay G, Colombo G, Concha Á, Graña B, Figueroa A: Heat Shock Protein 90 Chaperone Regulates the E3 Ubiquitin-Ligase Hakai Protein Stability. CANCERS 2020, 12(1):215.

24. Li J, He J, Wang Y, Shu Y, Zhou J: SMC1 promotes proliferation and inhibits apoptosis through the NF-kB signaling pathway in colorectal cancer. ONCOL REP 2019, 42(4):1329-1342. 
25. Lidza, Kalifa, Jennifer, S., Gewandter, Rhonda, J., Staversky, Elaine, A.: Kalifa L , Gewandter J S , Staversky R J , et al. DNA double-strand breaks activate ATM independent of mitochondrial dysfunction in A549 cells. FREE RADICAL BIO MED 2014(75:30-39).

26. Jackson SP, Bartek J: The DNA-damage response in human biology and disease. NATURE 2009, 461(7267):1071-1078.

27. Kory N, Wyant GA, Prakash G, Uit De Bos J, Bottanelli F, Pacold ME, Chan SH, Lewis CA, Wang T, Keys HR et al: SFXN1 is a mitochondrial serine transporter required for one-carbon metabolism. SCIENCE 2018, 362(6416):t9528.

28. Zhang Y, Zhang X, Fleming MR, Amiri A, El-Hassar L, Surguchev AA, Hyland C, Jenkins DP, Desai R, Brown MR: Kv3.3 Channels Bind Hax-1 and Arp2/3 to Assemble a Stable Local Actin Network that Regulates Channel Gating. CELL 2016:S1633427006.

29. Zhang Y, Wang P, Zhang Q, Yao X, Yao S: elF3i activity is critical for endothelial cells in tumor induced angiogenesis through regulating VEGFR and ERK translation. Oncotarget 2017, 8(12).

30. Jefferson M, Bone B, Buck JL, Powell PP: The Autophagy Protein ATG16L1 Is Required for Sindbis Virus-Induced elF2a Phosphorylation and Stress Granule Formation. Viruses 2020, 12(1):39.

31. Pardal AJ, Fernandes-Duarte F, Bowman AJ: The histone chaperoning pathway: from ribosome to nucleosome. ESSAYS BIOCHEM 2019, 63(1):29-43.

32. Sawan C, Herceg Z: Histone Modifications and Cancer. ADV GENET 2010, 70:57-85.

33. Nagelkerke A, Span PN: Staining Against Phospho-H2AX ( $\mathrm{H}-\mathrm{H} 2 \mathrm{AX})$ as a Marker for DNA Damage and Genomic Instability in Cancer Tissues and Cells: Springer International Publishing; 2016.

34. Gilson P, Drouot G, Witz A, Merlin J, Becuwe P, Harlé A: Emerging Roles of DDB2 in Cancer. INT J MOL SC/2019, 20(20):5168.

35. Nougayrède JP, Foster GH, Donnenberg MS: Enteropathogenic Escherichia coli effector EspF interacts with host protein Abcf2. CELL MICROBIOL 2007, 9(3):680-693.

36. Hardwidge PR, Rodriguez-Escudero I, Goode D, Donohoe S, Eng J, Goodlett DR, Aebersold R, Finlay BB: Proteomic Analysis of the Intestinal Epithelial Cell Response to Enteropathogenic Escherichia coli. J BIOL CHEM 2004, 279(19):20127-20136.

37. Dean P, Scott JA, Knox AA, Quitard S, Watkins NJ, Kenny B: The enteropathogenic E. coli effector EspF targets and disrupts the nucleolus by a process regulated by mitochondrial dysfunction. PLOS PATHOG 2010, 6(6):e1000961.

38. Maddocks ODK, Short AJ, Donnenberg MS, Bader S, Harrison DJ: Attaching and Effacing Escherichia coli Downregulate DNA Mismatch Repair Protein In Vitro and Are Associated with Colorectal Adenocarcinomas in Humans. PLOS ONE 2009, 4.

39. Maddocks ODK, Scanlon KM, Donnenberg MS: An Escherichia coli Effector Protein Promotes Host Mutation via Depletion of DNA Mismatch Repair Proteins. MBIO 2013, 4(3):e113-e152.

40. Luo Y, Deng X, Cheng F, Li Y, Qiu J: SMC1-Mediated Intra-S-Phase Arrest Facilitates Bocavirus DNA Replication. J VIROL 2013, 87(7):4017-4032.

41. Ying Z, Fei Y, Liang W, Zhuo W, Naijin Z, Zhijun W, Ziwei L, Xiaoyu S, Shi W, Liu C: Phosphorylation of SMC1A promotes hepatocellular carcinoma cell proliferation and migration. INT J BIOL SCI 2018, 14(9):1081-1089.

42. Kipanyula MJ, Etet PFS, Vecchio L, Farahna M, Nukenine EN, Kamdje AHN: Signaling pathways bridging microbial-triggered inflammation and cancer. CELL SIGNAL 2013, 25(2):403-416.

43. Wang X, Du Y, Hua Y, Fu M, Niu C, Zhang B, Zhao W, Zhang Q, Wan C: The EspF N-Terminal of Enterohemorrhagic Escherichia coli 0157:H7 EDL933w Imparts Stronger Toxicity Effects on HT-29 Cells than the C-Terminal. FRONT CELL INFECT MI 2017, 7.

\section{Figures}

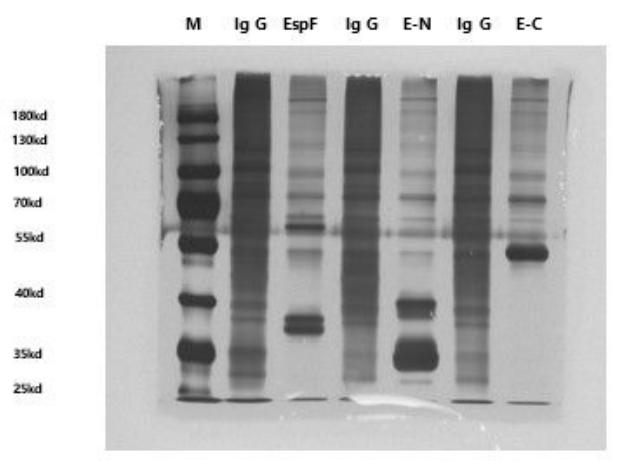

Figure 1

Silver staining of proteins. From left to right is the Maker, pEGFP-EspF control group and experimental group, the pEGFP-EspF / $\mathrm{N}$ control group and experimental group, and the pEGFP-EspF / C plasmid group. Plasmids were transfected into 293T cells for $48 \mathrm{~h}$. The proteins were lysed electroporated on an SDS-PAGE gel. The pEGFP-EspF group had two differential bands at 35-40 kDa, the pEGFP-EspF / $\mathrm{N}$ group had a differential band at $40 \mathrm{kDa}$, and the pEGFPEspF / C group had a differential band at $130 \mathrm{kDa}$. 
A

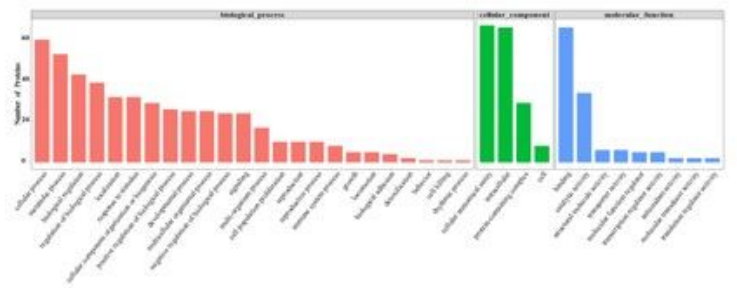

B
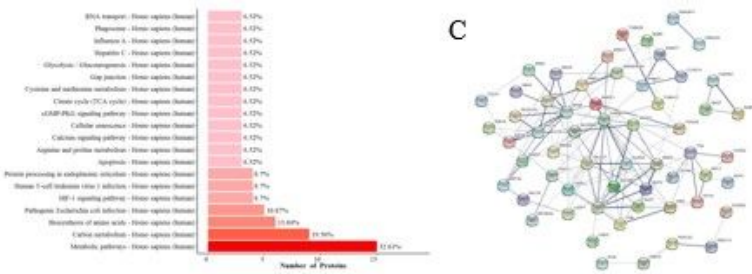

Figure 2

GO enrichment, KEGG pathway, and STRING analyses of the $38 \mathrm{kDa}$ proteins interacting with EspF. (A) GO annotation of identified EspF-interacting proteins in biological processes, cell components, and molecular functions. (B) The distribution of the top 20 interacting protein of Genes and Genomes (KEGG) pathways. (C) STRING analysis of statistically significant proteins associated with the EspF-interacting proteins. The networks also illustrate the functional relationships (the edges) between the nodes; the thickness of the nodes is directly proportional to the association's significance score.

A

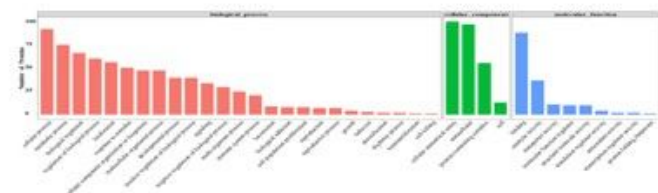

B

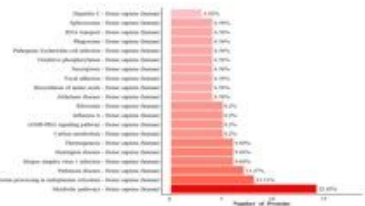

C

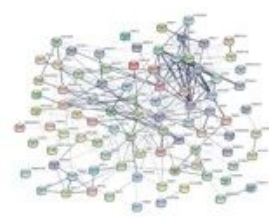

Figure 3

GO enrichment, KEGG pathway, and STRING analyses of the $36 \mathrm{kDa}$ proteins interacting with EspF. (A) GO annotation of identified EspF-interacting proteins in BPs, CCs, and MFs. (B) The distribution of the top 20 interacting proteins of Genes and Genomes (KEGG) pathways. (C) STRING analysis of statistically significant proteins detected in EspF-interacting proteins. The networks also illustrate the functional relationships (the edges) between the nodes, the thickness of which is directly proportional to the association's significance score.

A

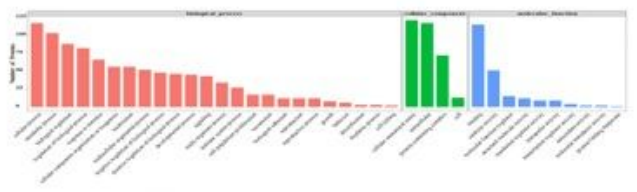

B

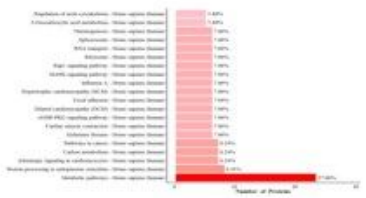

C

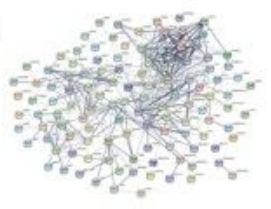

Figure 4

GO enrichment, KEGG pathways, and STRING analyses of the $41 \mathrm{kDa}$ EspF-N terminus interacting proteins. (A) GO annotation of identified EspF-N terminus interacting proteins in BPs, CCs, and MFs. (B) The distribution of the top 20 interacting proteins in Genes and Genomes (KEGG) pathways. (C) STRING analysis of statistically significant proteins detected in EspF-N-interacting proteins. The networks also illustrate the functional relationships (the edges) between the nodes, the thickness of which is directly proportional to the association's significance score. 
A

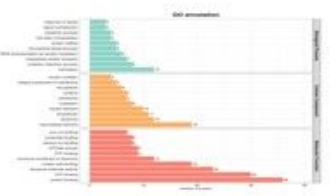

C

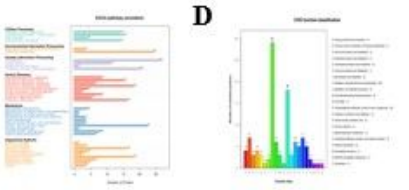

B

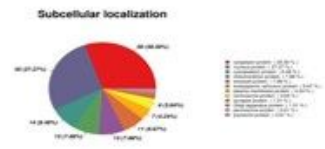

$\mathbf{E}$

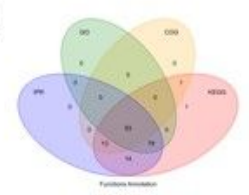

Figure 5

GO enrichment, KEGG pathways, and STRING analyses of the $130 \mathrm{kDa}$ EspF-C terminus interacting proteins. (A) GO annotation of the identified EspF-Cinteracting proteins in BPs, CCs, and MFs. (B) Subcellular location of the interacting protein. (C) The distribution of the interacting protein in Genes and Genomes (KEGG) pathways. (D) The COG function classification of the interacting protein (COG) pathways. (E) A Venn diagram of common proteins found in the above bioinformatics analysis.
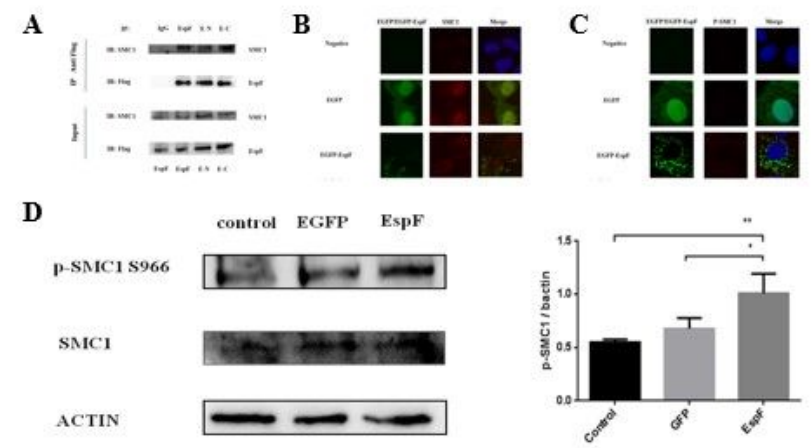

E
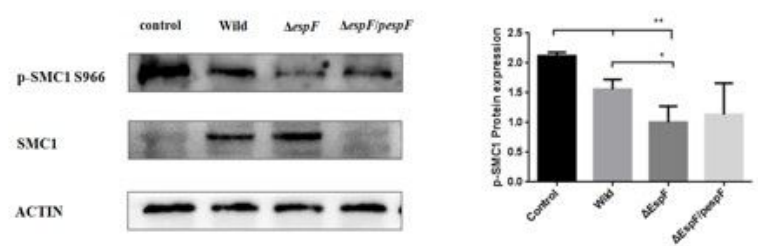

\section{Figure 6}

EspF interacts with SMC1 and can increase its phosphorylation level. (A) Co-immunoprecipitation to identify the interactions between SMC1 and EspF/EspF-N /EspF-C. The pEGFP-EspF, pEGFP-EspF-N, and pEGFP-EspF-C were tagged with 3xFLAG and were transfected into 293T cells. Cell proteins were extracted and incubated with Flag/lgG agarose beads. Then, the cell proteins (input) and the beads binding proteins were prepared for 10\% SDS-PAGE. (B) Immunofluorescence to identify the interactions between EspF and SMC1. pEGFP-N1 and pEGFP-EspF were transfected into Vero cells. Then, the cells were incubated with anti-SMC1 antibody, and the colocalization was observed under a confocal microscope. The arrow points to co-location. (C) Immunofluorescence to identify the p-SMC1 protein expression. pEGFP-N1 and pEGFP-EspF were transfected into Vero cells. Then, the cells were incubated with anti-p-SMC1 antibody, and the colocalization was observed under a confocal microscope. (D) Western blot to identify p-SMC1 protein expression in Caco2 2 cells. pEGFP-N1 and pEGFP-EspF were transfected into Caco2 cells. Then, the cells were lysed for Western blot. The p-SMC1 protein expression index as determined by statistical analysis. ${ }^{*} \mathrm{P}<0.05$, ${ }^{*} \mathrm{P}<0.01$. (E) Western blot to identify $\mathrm{p}$-SMC1 protein expression in $\mathrm{HT}-29 \mathrm{cells}$. Wild, $\Delta$ espF, and $\Delta$ espF /pespF strains infected HT-29 cells for six hours, and then the cells were lysed for western blot. 

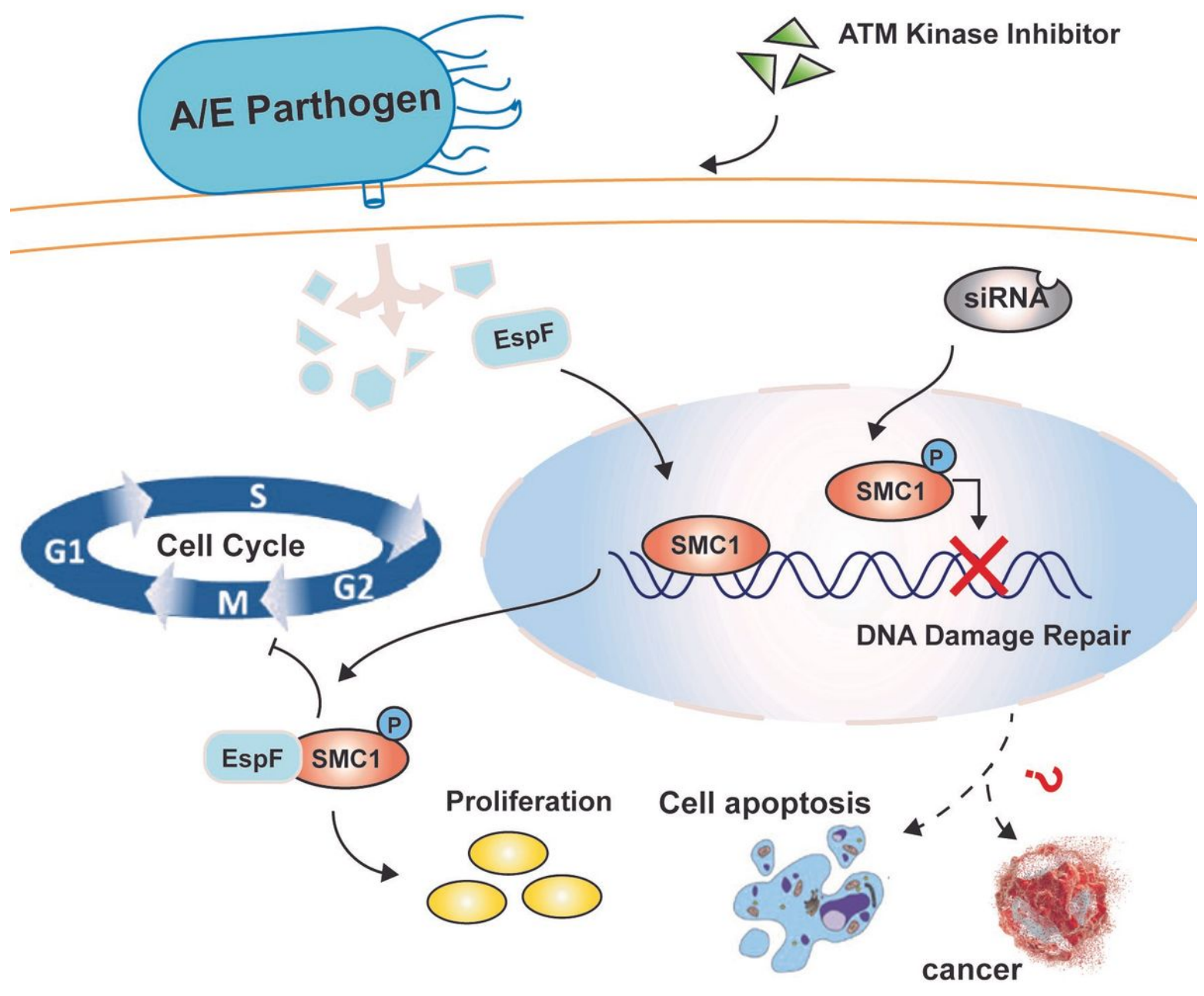

Figure 7

What is the biological effect of EspF mediated by SMC1? EspF can increase the expression of p-SMC1 in Caco2 cells, and relocalize it in the cytoplasm. Therefore, EspF may mediate DNA damage, cell proliferation, cell cycle arrested, cell apoptosis, and even cancer by interacting with SMC1.

\section{Supplementary Files}

This is a list of supplementary files associated with this preprint. Click to download.

- MSresultsofEspFN40kDaGOanalysis.png

- KEGG.anno.pdf

- IgGusagedata.txt

- KEGG.anno.ko2protein.xls

- MSresultsofEspF36kDaGOanalysis.png

- IPR.anno.png

- 2.6.statREADME.txt

- IPR.anno.ipr2protein.xls

- Pathwayresults.xlsx

- lenprot.png

- lenprot.pdf

- KEGG.anno.png

- GOanalysis.xIsx

- 2.5.SubcellarREADME.txt

- IPR.anno.pdf

- MSresultsofEspFN40kDa.xlsx 
- IPR.anno.xls

- Flagusagedata.txt

- GOKEGGCOG.anno.xls

- IgGmodification.xlsx

- MSresultsofEspF38kDaGOanalysis.png

- MSresultsofEspF38kDapercent.png

- MSresultsofEspF38kDapercent.pdf

- Flagmodification.xlsx

- Subcellular.localizationanno.xls

- MSresultsofEspF38kDaGOanalysis.pdf

- KEGG.anno.xls

- ppi.xlsx

- quality.xls

- MSresultsofEspF36kDapercent.png

- SMC1.txt

- MSresultsofEspF36kDaGOanalysis.pdf

- MSresultsofEspF36kDapercent.pdf

- Peppro.xlsx

- 3.ProtExprQuantificationREADME.txt

- GO.anno.png

- dmassdistri.pdf

- MSresultsofEspF36kDa.png

- MSresultsofEspFN40kDaGOanalysis.pdf

- MSresultsofEspF38kDa.pdf

- MSresultsofEspF36kDa.pdf

- Subcellular.localization.pdf

- stat.venn.png

- MWdistri.pdf

- MWdistri.png

- MSresultsofEspF38kDa.png

- allsample.xls

- Subcellular.localization.png

- uniqdistri.png

- uniqdistri.pdf

- MSresultsofEspFN40kDapercent.pdf

- MSresultsofEspFN40kDa.pdf

- MSresultsofEspFN40kDa.png

- stat.venn.svg

- 1.datacontrolREADME.txt

- 2.3.KEGGREADME.txt

- 2.2.COGREADME.txt

- dmassdistri.png

- 2.4.IPRREADME.txt

- Go.anno.xls

- GO.anno.pdf

- MSresultsofEspF38kDa.xlsx

- covprot.pdf

- covprot.png

- 2.1.GOREADME.txt

- COG.anno.cog2protein.xls

- GO.anno.go2protein.xls

- MSresultsofEspF36kDa.xIsx 
- COG.anno.xls

- COG.anno.png

- allsample.fa

- COG.anno.pdf

- MSresultsofEspFN40kDapercent.png

Page 15/15 